\title{
Early experience in ureteroscopy for the management of ureteric stone
}

\author{
A.Agarwal ${ }^{1}$, P.Shrestha ${ }^{2}$, W.K.Belokar ${ }^{3}$
}

${ }^{1}$ Mch Resident ${ }^{3}$ Professor and HOD, Department of Urology, ${ }^{2}$ Resident, Department of Surgery, CMS-TH Bharatpur, Nepal

\begin{abstract}
Ureteroscopy has revolunized the management of ureteric calculus and it has become the need of every urology center today. The purpose of this study is to report our experience and results in the management of ureteric calculus by semi-rigid ureteroscopy and pneumatic lithotripsy and also to access the safety of procedure in untrained hands.

Retrospective analysis of 48 consecutive patients of ureteric calculus treated by semi-rigid ureteroscopy and pneumatic lithotripsy between June 2010 and October 2010 in urology unit, College of Medical Sciences, Bharatpur, Nepal was done.

34 female patients (70.8\%) and 14 male patients (29.2\%) were identified. Mean age of female was 30.9years (range 17 to 45 years) and that of male was 46.4 years (range 33 to 63years). Mean stone size in female was $8.91 \mathrm{~mm}$ (range 6.5 to $11.2 \mathrm{~mm}$ ) and in male it was $9.11 \mathrm{~mm}$ (range 6 to $14.2 \mathrm{~mm}$ ). Lower ureteric calculus was present in 29 and 8(77.1\%), mid ureteric calculus in 2 and 2(8.3\%) and upper ureteric calculus in 3 and $4(14.6 \%)$ female and male patients respectively. Ureteric perforation occurred in 2 patients (4.2\%). Ureteric avulsion occured in 1 female patient $(2.1 \%)$ with upper ureteric calculus. DJ stent was keept in 34(70.8\%) cases but in 11 cases (32.6\%) DJ stent had to be removed earlier than 4 weeks due to stent related problems. Urine culture was sent before ureteroscopy in all the patients and was sterile in 24 patients (50\%), contaminated growth in 12 patients $(25 \%)$, grew E-coli in 8 patients (16.7\%) and Kliebsella pneumonia in 2 patients(4.2\%). Average duration of stay was 2.7 days. 5 patients developed fever post operatively, managed by intravenous antibiotics. There was no mortality.

Though ureteroscopy has become a boon for the management of ureteric calculus, complications like perforation, ureteric avulsion, residual stone and stone migration are more in upper ureteric calculus and stone size $>1 \mathrm{~cm}$. Stenosed ureteral assess can be made with proper guide wire selection and prior dilatation .The procedure is relatively safe even in inexperienced hands.
\end{abstract}

Key words: Pneumatic, calculus, lithotripsy, ureter, ureteroscopy, experience.

\section{Introduction}

The first ureteroscopy was performed in 1912 by Hugh Hampton Young in a patient with posterior Correspondence: A. Agarwal

E-mail: draaman@gmail.com urethral valves when a rigid cystoscope was advanced into the dilated ureter. Initially, all ureteroscopes were rigid in design and consisted of a rod-lens system with outer diameters ranging 
A.Agarwal et al, Early experience in ureteroscopy for the management of ureteric stone

from 12 to 13.5 Fr. By the mid-1980s, rigid endoscopes were reduced to $8.5 \mathrm{Fr}$ in diameter and contained a working channel. ${ }^{1,2}$ It was not until 1989 that the next generation of "semirigid" ureteroscopes was developed and contained fiberoptics rather than a rod-lens system, ${ }^{3}$ and in 1993 the current generation of smaller endoscopes was available for use. ${ }^{4}$

Semirigid ureteroscopes typically have a tapered distal tip (e.g., 6.75 to $9.0 \mathrm{Fr}$ ) that dilates to a larger diameter shaft closer to the eyepiece (e.g., 8.4 to $10.1 \mathrm{Fr})$. The advantages of the semirigid ureteroscope include a large working channel, faster irrigation flow, and a larger field of view because of the larger number of fiberoptic bundles compared with the corresponding flexible instruments. Semirigid ureteroscopes are typically used for treatment of ureteral pathology below the iliac vessels but may be utilized above the iliac vessels especially in female patients. In males because of longer urethras it is often difficult to use the semirigid ureteroscope above the iliac vessels.

Ureteroscopy has become a standard urologic technique and is used in a wide variety of situations for diagnosis and treatment. The main use for the technique of supravesical endoscopy is in the treatment of urolithiasis. Other therapeutic indications are endoureterotomy for ureteral stricture, retrograde endopyelotomy for ureteropelvic junction obstruction, biopsy/ablation of upper tract transitional cell carcinoma (TCC) and retrieval of migrated ureteral stent. Ureteroscopy now a days is being increasingly used for diagnostic evaluation of positive cytology with normal cystoscopy, monitoring of previous upper tract TCC, Evaluation of filling defects on intravenous pyelography/retrograde pyelography and Undiagnosed gross hematuria The advent of smaller semirigid and flexible fiberoptic endoscopes has allowed routine retrograde access to the proximal ureter and kidney, and when combined with the holmium:yttrium-aluminum-garnet (YAG) laser, provides a safe and highly effective retrograde method of intracorporeal lithotripsy. The current generation of flexible, actively deflectable fiberoptic endoscopes makes virtually every part of the kidney, including the lower pole, accessible for the treatment of calculi. The purpose of this study was to report our experience

and results in the management of ureteric calculus by semirigid ureteroscope and to assess the safety of the procedure in inexperienced hands.

\section{Materials and methods}

The medical records of all those patients treated for ureteric calculus between June 2010 and October 2010 were reviewed retrospectively at urology unit, College of Medical Sciences, Bharatpur, Nepal. Patients' age, stone size, stone position, upper urinary tract changes, duration of hospital stay, treatment and outcome were recorded and reviewed in the light of literature.

\section{Results}

In our study we analyzed 48 patients who were diagnosed and treated for ureteric stones, out of which 34 patients $(70.8 \%)$ were female and 14 patients $(29.2 \%)$ were male. Mean age of female 
was 30.9years (range 17 to 45 years) and that of male was 46.4 years (range 33 to 63years). Mean stone size in female was $8.91 \mathrm{~mm}$ (range 6.5 to $11.2 \mathrm{~mm}$ ) and in male it was $9.11 \mathrm{~mm}$ (range 6 to $14.2 \mathrm{~mm})$. Lower ureteric calculus was present in 29 and $8(77.1 \%)$, mid ureteric calculus in 2 and $2(8.3 \%)$ and upper ureteric calculus in 3 and $4(14.6 \%)$ female and male patients respectively (chart 1). Ureteric perforation occurred in 2 male(4.2\%) patients both in upper ureteric calculus, managed by double-J (DJ) stenting and in 1 female $(2.1 \%)$ patient with upper ureteric calculus ureteric avulsion occurred just below pelvic and ureteric junction managed by Boari's flap and mobilizing the kidney. DJ stent was keept in $34(70.8 \%)$ out of 48 patients following ureteroscopy. In 11 patients $(32.6 \%)$ (chart3) DJ stent had to be removed earlier than 3 weeks due to stent related problems like pain, fever, dysuria, frequency, etc. Stents used were 26F, both side open and Rompsons company in all the patients. None of the patient was found to be either hypercalcemic or hyperuricemic. Urine culture was sent before ureteroscopy in all the patients and was sterile in 24 patients $(50 \%)$, contaminated growth in 12 patients(25\%), grew E-coli in 8 patients(16.7\%) and Klebsiella pneumonia in 2 patients $(4.2 \%)$ (chart 2). Stone migration to kidney occurred in 3 patients. All three stones lodged into lower pole of kidney and patients had to subjected for Shock Wave Lithotripsy (SWL) for stone clearance (chart4). Two patients did not follow up with culture report. Average duration of stay was 2.7 days. 5 patients developed fever post operatively, managed by intravenous antibiotics There was no mortality.

\section{Discussion}

The 1997 AUA panel guidelines recommended ureteroscopy or shockwave lithotripsy as acceptable treatment for stones in the distal ureter that are unlikely to pass spontaneously or cause significant symptoms requiring multiple hospital visits. ${ }^{5}$ Stonefree rates in the distal ureter exceed $95 \%$ after ureteroscopy ${ }^{6}$ and $75 \%$ to $97 \%$ after SWL. ${ }^{7}$ Ureteroscopy may produce consistently higher stone-free rates but is more invasive than SWL. Furthermore, SWL is likely to require more than one session, ancillary treatments, and is not as widely available as ureteroscopy in some healthcare settings. For distal ureteral stones larger than $1 \mathrm{~cm}$, ureteroscopy and intracorporeal lithotripsy decrease the need for ancillary treatment and typically render the patient stone free following one treat-ment compared with SWL. Studies comparing SWL with ureteroscopy for distal stones demonstrate that patients treated with ureteroscopy not only had higher stone-free rates but also required less operating room time, less fluoroscopy time, and less time to achieve a stone-free status. ${ }^{8}$ Both ureteroscopy and SWL are viable options in the treatment of distal ureteral stones, and the decision on which treatment to employ should be based on patients' preferences and the availability of each modality. ${ }^{5}$

AUA panel guidelines recommend SWL as firstline therapy for proximal ureteral stones smaller than $1 \mathrm{~cm}$. Ureteroscopy and percutaneous nephrolithotomy are acceptable choices for failed 
A.Agarwal et al, Early experience in ureteroscopy for the management of ureteric stone

SWL. ${ }^{9}$ For proximal ureteral stones larger than 1

$\mathrm{cm}$, SWL, percutaneous nephrolithotomy, and ureteroscopy are all equally viable options according to AUA panel guidelines. ${ }^{9}$ Patients with stones larger than $1 \mathrm{~cm}$ in the proximal ureter treated by ureteroscopy have excellent stone-free rates ranging from $92 \%$ to $97 \% .^{10}$

Ureteroscopy for intrarenal stones is very effective and produces stone-free rates ranging from $84 \%$ to $92 \% .{ }^{11}$ Intrarenal stones smaller than $2 \mathrm{~cm}$ may be successfully treated ureteroscopically while larger stones $(>2 \mathrm{~cm})$ are better managed with a percutaneous approach. Success rates of $85 \%$ have been reported for lower pole stones treated ureteroscopically. ${ }^{12}$

Complications of ureteroscopy include intraoperative failure to access (ureter, kidney, or stone), failure to fragment the stone, stone migration into the ureteral wall, mucosal trauma, ureteral perforation, ureteral avulsion early postoperative gross hematuria, renal colic (small residual stone fragments, blood clot, or ureteral edema), large residual stone fragments (that require ancillary procedures), pyelonephritis, urinoma, ureteral stent symptoms, late postoperative ureteral stricture and forgotten encrusted ureteral stent.

\section{Conclusion}

Ureteroscopy is a mainstay in the treatment of urologic stone disease. It provides an effective method of lithotripsy with stone-free rates in excess of $80 \%$ even in inexperienced hands and can also be used for the diagnosis and treatment of upper tract transitional cell cancer, ureteral stricture, and ureteropelvic junction obstruction. Complications related to ureteroscopy decreases with increase in exprences and proper case selection. Most common problem we faced in early stage was unability to assess through narrow ureteric orifices. With experience, proper guide wire selection and prior balloon dilatation, now, any ureteric orifice is accessable.

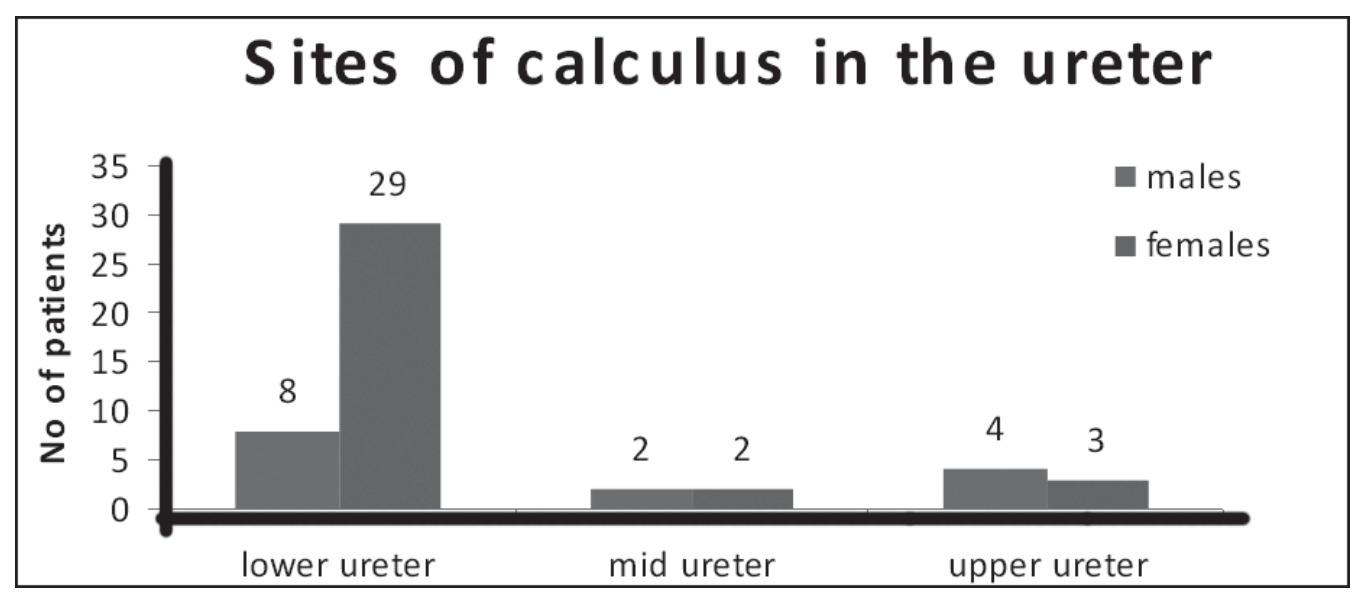

Chart 1. Sites of calculus in the ureter 
Journal of College of Medical Sciences-Nepal, 2011, Vol-7, No-4

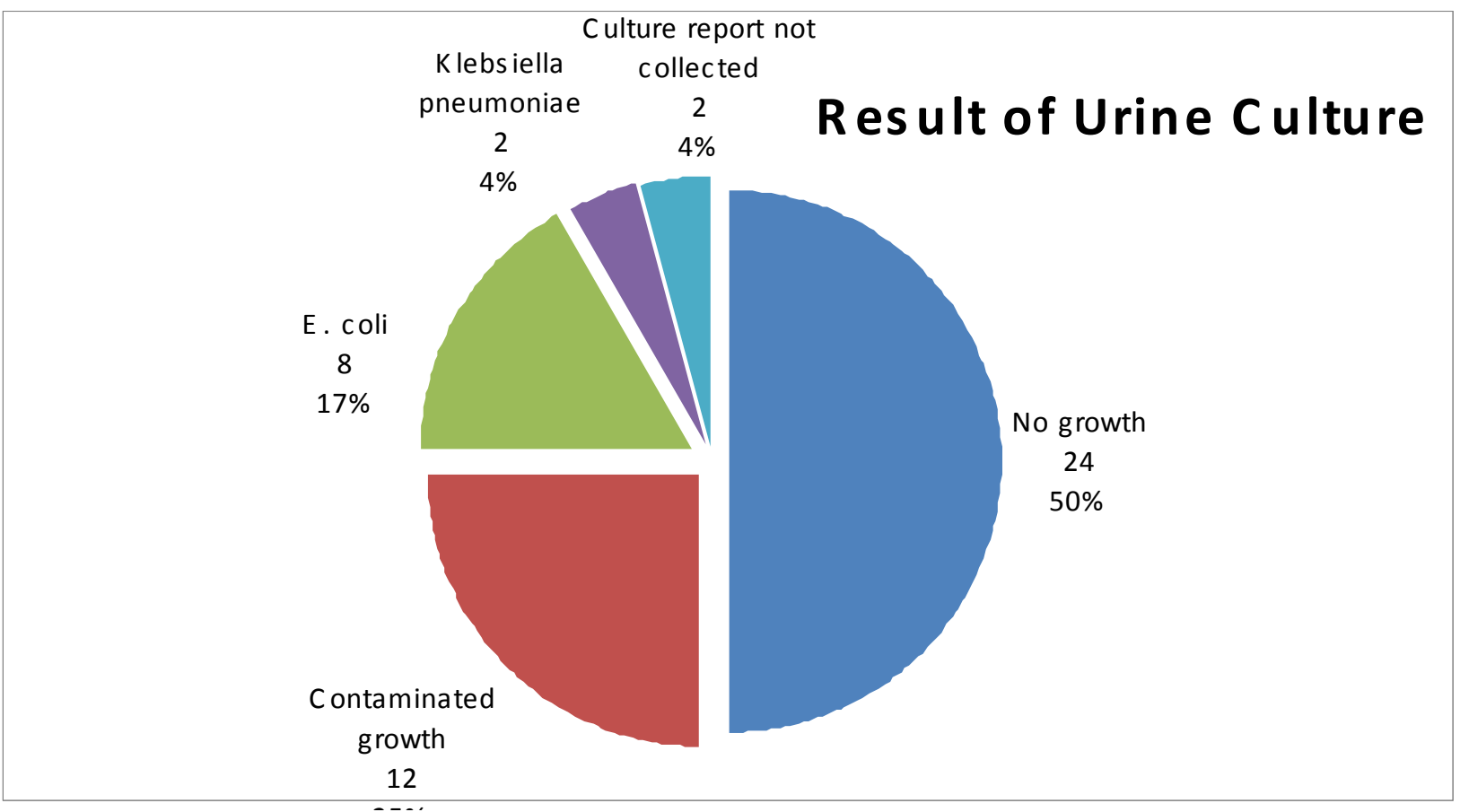

Chart 2. Result of Urine Culture

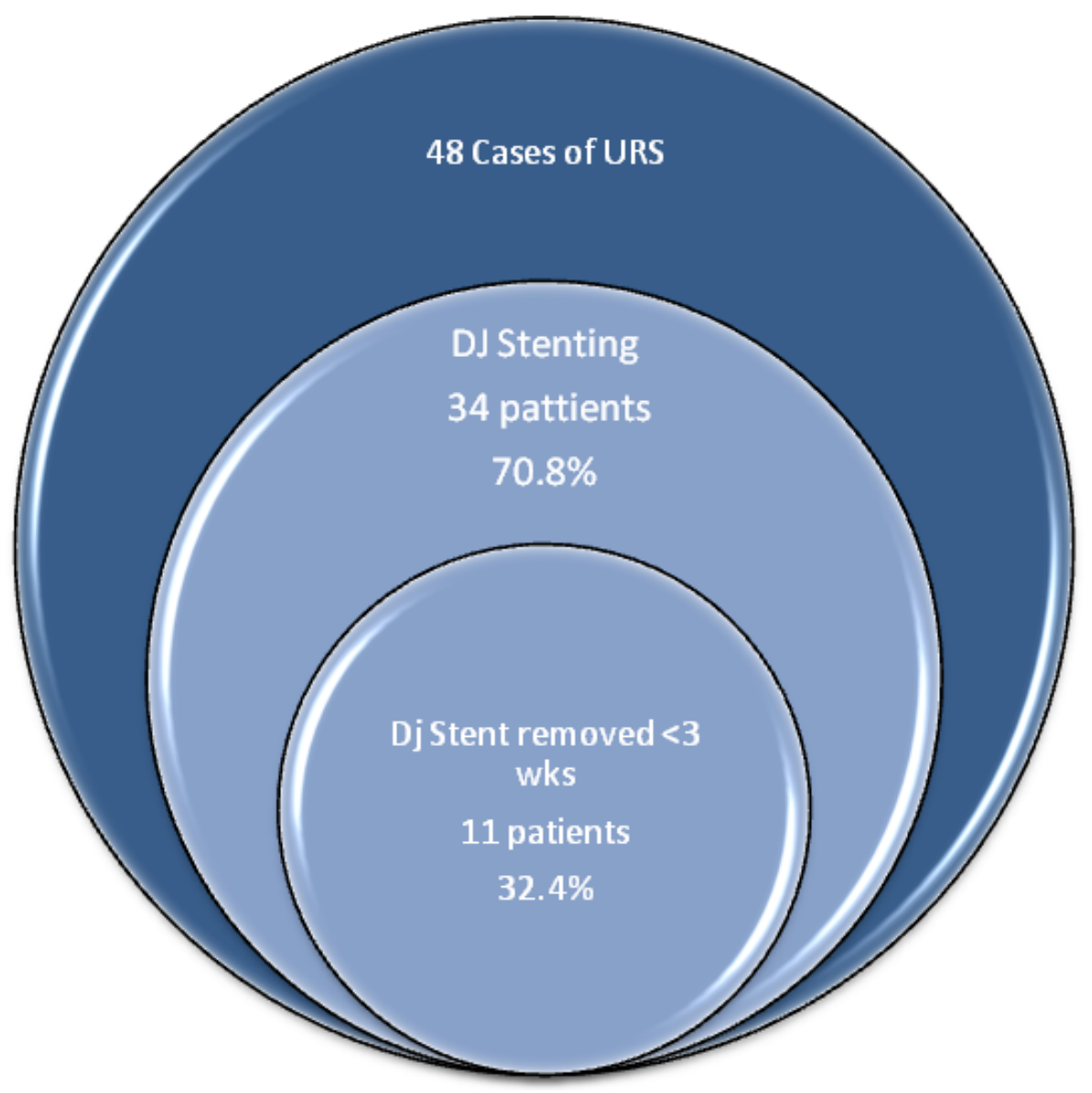

Chart 3: DJ stenting following Ureterorenoscopy 


\section{Total Cases}

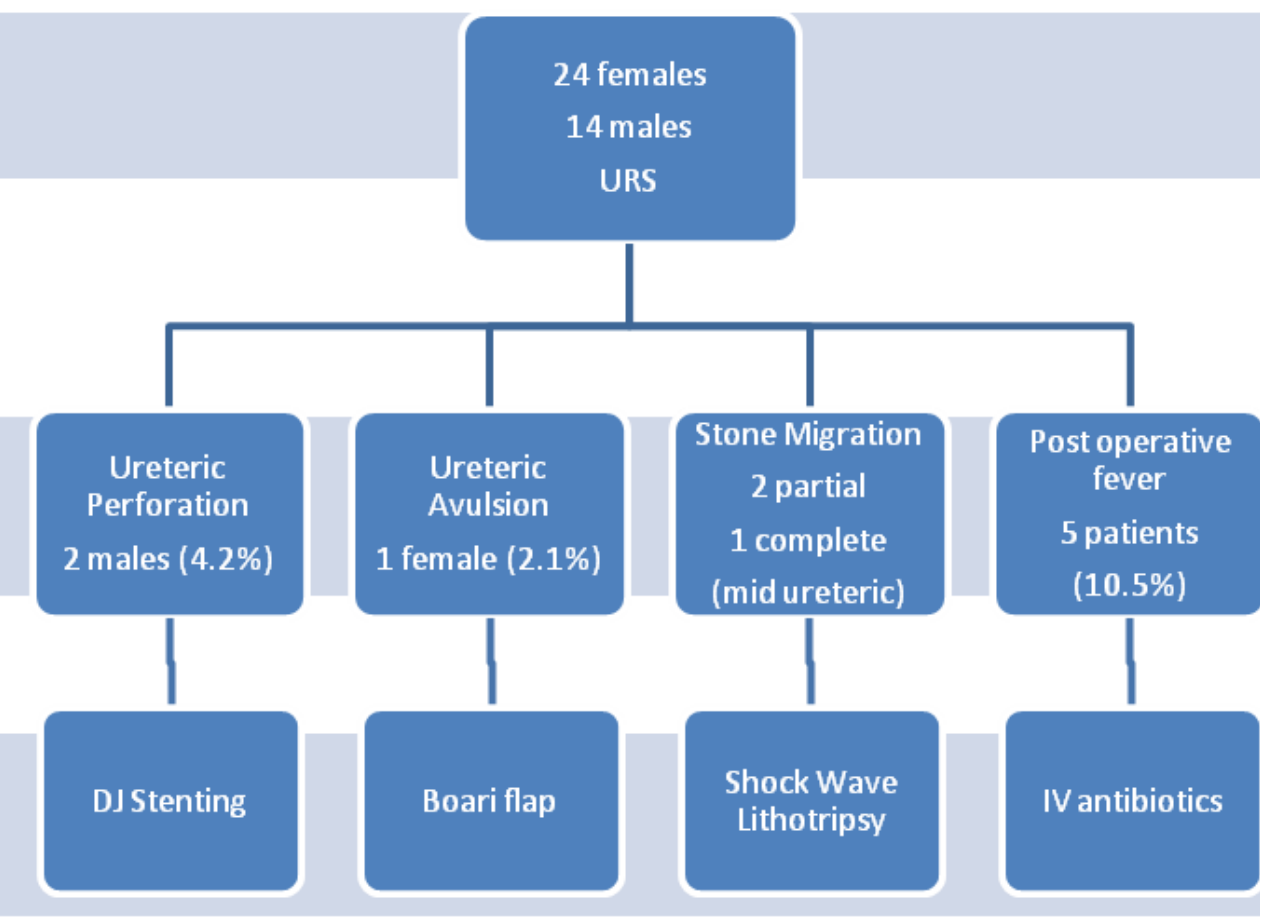

\section{Chart 4: Complications and their management}

\section{References}

1. J.L.Huffman. Experience with the 8.5 French compact rigid ureteroscope. Semin Urol 1989a;7:3-6.

2. J.L.Huffman. Early experience with the $8.5 \mathrm{~F}$ compact ureteroscope. Surg Endosc 1989b;3:164-6.

3. S.P.Dretler, G.Cho: Semirigid ureteroscopy: A new genre. J Urol 1989; 141:1314-6

4. O.Abdel-Razzak, D.H.Bagley. The $6.9 \mathrm{~F}$ semirigid ureteroscope in clinical use. Urology1993;41:45-8.

5. J.W.Segura, G.M.Preminger, D.G.Assimos, et al. Ureteral Stones Clinical Guidelines Panel summary report on the management of ureteral calculi. The American Urological Association. $J$ Urol 1997;158:1915-21.

6. M.Sofer, J.D.Watterson, T.A.Wollin, et al. Holmium:YAG laser lithotripsy for upper urinary tract calculi in 598 patients. J Urol 2002;167:31-4.

7. M.S.Pearle, R.Nadler, E.Bercowsky, et al: Prospective randomized trial comparing shock wave lithotripsy and ureteroscopy for management of distal ureteral calculi. J Urol 2001;166:1255-60.

8. S.Hautmann, M.G.Friedrich, S.Fernandez, et al. Extracorporeal shockwave lithotripsy compared with ureteroscopy for the removal of small distal ureteral stones. Urol Int 2004;73:238-43.

9. A.J. wein, L.R Kavoussi et al, ureteroscopy and retrograde ureteral access, Campbell-Walsh Urology, $9^{\text {th }}$ edi.Saunders, Elsevier.2007:1518-9

10. C.F.Wu, J.J.Shee, W.Y.Lin, et al. Comparison between extracorporeal shock wave lithotripsy and semirigid ureterorenoscope with holmium:YAG laser lithotripsy for treating large proximal ureteral stones. $J$ Urol 2004;172:1899-902.

11. G.K.Chow, D.E.Patterson, M.L.Blute, et al. Ureteroscopy: Effect of technology and technique on clinical practice. J Urol 2003;170:99-102.

12. M.Grasso, Y.Chalik. Principles and applications of laser lithotripsy: Experience with the holmium laser lithotrite. J Clin Laser Med Surg 1998;16:3-7. 